\title{
Current perspectives on genotype classification and individualized drug targeting in triple-negative breast cancer
}

\author{
Xiao-tian Ma ${ }^{1}$, Shou-hua Rong ${ }^{1}$, Yu-chao Zhang ${ }^{2}$, Li-ting Jia ${ }^{1 *}$ \\ ${ }^{1}$ Clinical Laboratory, ${ }^{2}$ Reproductive Center, The Third Affiliated Hospital to Zhengzhou University, Zhengzhou 450052, China
}

${ }^{\star}$ For correspondence: Email: kfyxgcxq21367@163.com

Sent for review: 8 December 2017

Revised accepted: 28 January 2018

\begin{abstract}
Triple negative breast cancer (TNBC), a special subset of breast cancer, refers to negative expressions of estrogen receptors (ER), progesterone receptors (PR) and human epidermal growth receptor 2 (HER2). It is associated with extreme local recurrence and distant metastasis with highly invasive character. With advances in genomics, the bases of molecular classification of TNBC now include the heterogeneity of its expression at the molecular level and clinical pathology, apart from classical immunohistochemistry. Every subtype of TNBC has different individualized target drugs, which include epidermal growth factor receptor (EGFR) inhibitor, poly-AD-ribose polymerase (PARP) inhibitor, anthracycline or paclitaxel, immunotherapy and vascular endothelial growth factor receptor (VEGFR) inhibitor. Combinations of target drugs are also used. Thus, there are no widely recognized standards of genotype classification and individualized drug targeting in TNBC. In this review, relevant studies and latest developments on TNBC are presented.
\end{abstract}

Keywords: Triple-negative breast cancer, Genotype classification, Individualized drug targeting, Breast cancer

\begin{abstract}
This is an Open Access article that uses a funding model which does not charge readers or their institutions for access and distributed under the terms of the Creative Commons Attribution License (http://creativecommons.org/licenses/by/4.0) and the Budapest Open Access Initiative (http://www.budapestopenaccessinitiative.org/read), which permit unrestricted use, distribution, and reproduction in any medium, provided the original work is properly credited.
\end{abstract}

Tropical Journal of Pharmaceutical Research is indexed by Science Citation Index (SciSearch), Scopus, International Pharmaceutical Abstract, Chemical Abstracts, Embase, Index Copernicus, EBSCO, African Index Medicus, JournalSeek, Journal Citation Reports/Science Edition, Directory of Open Access Journals (DOAJ), African Journal Online, Bioline International, Open-J-Gate and Pharmacy Abstracts

\section{INTRODUCTION}

Evidence has shown that breast cancer is widely considered as a leading cause of death among women, and it has been associated with significantly increasing morbidity in recent years [1]. In triple-negative breast cancer (TNBC), a subtype of breast cancer which is about $15 \%$ of breast cancers, ERs, PR, and HER2 are not expressed $[2,3]$. Due to its poor prognosis, high local recurrence rate and distant metastasis,
TNBC does not respond well to targeted therapies [4].

This disease is a highly heterogeneous tumor which has been classified into 6 subtypes according to histology and molecular biology characteristics; different molecular subtypes of TNBC have differences in clinical expressions, responses to treatment and prognosis [4-6]. Chemotherapy is currently a major systemic treatment scheme for TNBC. However, although cytotoxic chemotherapeutic agents for TNBC 
patients are supposed to be the effective therapy option, some patients are inherently in sensitive to cytotoxic chemotherapy, while many patients have shown drug resistance, resulting in disease recurrence $[7,8]$.

Previous reports indicated that in TNBC, the different expressions of genic subtypes produce different hereditary events. For example, mutations in RAD51D, MRE11 and PALB2 may confer a higher risk of TNBC in the subtypes [9]. Moreover, it has been reported that the high BRCA1 rs80350973 mutation associated with Ki67 indices might be a predictor of prognosis of TNBC [10]. Thus, these important genic hereditary events may provide critical guide for prediction of genic targeted therapies in TNBC.

The rapid developments in investigative technologies of tissue chip and tissue microarrays have facilitated studies on genotype classifications in TNBC, resulting in enhanced understanding and useful development of genic targeted therapies.

\section{HISTOPATHOLOGIC CHARACTERISTICS AND THERAPEUTIC ADVANCES OF TNBC}

\section{Genotype classification of triple-negative breast cancer}

In 2000, the utilization of tissue chip and microarrays from the report of Perou et al [11] led to the discovery of much greater differences in gene expressions between various breast cancers. On the basis of that finding, five basic subtypes of breast cancer were proposed, which include luminal A, luminal B, ERBB-2 (+), basallike and normal-like subtypes. A part of these subtypes pertains to triple-negative phenotype which was reported in 587 cases with TNBC by Lehmann et al [12]. Thus, the classification of TNBC was molecularly categorized as basal-like 1 (BL1) with the genic characteristics of cell cycle and DNA damage response, and basal-like 2 (BL2) subtypes with growth factor signaling and myo-epithelium marker; and immune-modulatory (IM) subtype. Others are mesenchymal (M) and mesenchymal stem-like (MSL) subtypes with expression of high differentiation and genes of growth factor pathways; and luminal androgen receptor (LAR) subtype activated by luminal signal [13].

Analysis of distinct molecular profiles by Burstein et al [14] identified the clinically-relevant TNBC subtypes as LAR, mesenchymal (MES), basallike immunosuppressed (BLIS), and basal-like immune-activated (BLIA), with each specific molecular target identified within the TNBC subtypes. Therefore, "triple-negative" is thought to refer to the common complexity of heterogeneous TNBC. Individualized drug targeting treatments related to heterogeneous TNBC and their clinical features are subjects of further studies.

\section{Individualized drug targeting in TNBC from genotype classification}

Due to lack of the expression of ER and HER2, TNBC is not sensitive to endocrine therapy and trastuzumab therapy. Thus, the patients have drug resistance, resulting in tumor recurrence, metastasis and poor prognosis. In addition, the signaling pathways, targets and molecular mechanisms of TNBC are too complex for easy development of individualized drug targeting.

\section{Poly ADP-ribose polymerase receptor inhibitor}

The members of poly ADP-ribose polymerase (PARP) PARP-1, PARP-2 and PARP-3 participate in each step of DNA damage repair [15]. When PARP inhibitor affected on cancer cells with BRCA1/2 mutations, the inhibition of PARP activity can prevent the formation, thus, the dependent PARP of DNA damage repair recombination were not resolved, at last, the DNA single-strand break (SSB) without repair led to the DNA stalled replication forks to convert the DNA double-strand breaks (DSBs), causing the apoptosis because of the homologous recombination (HR) lacked of $\mathrm{BRCA} 1 / 2$, which was called the "PARP-BRCA synthetic lethality" [16]. Meanwhile, the theory uncovered the mechanisms of PARP inhibitors intermediates selectively killed the HR defective cells to provide the epidemiological evidences.

Studies by Evans et al [17] found that patientderived xenografts (PDXs) harbored a heterogeneous set of genomic alterations consistent with the TNBC subtypes, which could capture the molecular and phenotypic heterogeneity of TNBC.

Thus, beyond germline BRCA1/2, the PARP inhibitor could alter tumors and cause tumor regression in a variety of molecular subtypes in TNBC. Clinical trials by Lee et al [18] showed the phase I/lb study of olaparib and carboplatin in 28 women with TNBC. With olaparib 400mg bid+carboplatin AUC4 as the maximum tolerated dose, the complete response (CR) was one patient $(69+$ months), while the partial response (PR) was 5 patients (median 4 months, range: 4 7 months). 
The clinical trial revealed that the combination of olaparib and carboplatin had modest activity in sporadic TNBC patients. However, the study was at phase $\mathrm{l} / \mathrm{lb}$, and the sample size was small. At present, there are a number of clinical experiments regarding combinations of PARP inhibitors with other chemotherapeutic drugs such as cisplatin, carboplatin and topotecans. The efficacy of combination treatment for BRCArelated breast cancer has attained approximately $70 \%$. However, the combination of olaparib and topotecan presented greater side effects in hematology [19-21].

\section{Platinum drugs}

Generally, platinum drugs can directly impact on DNA synthesis through the crosslinking of DNA which limits DNA unwinding and replication, resulting in anti-cancer effects. Studies by Bignon et al [22] showed pCR group and nonpCR patients with TNBC or deleterious BRCA1 or BRCA2 mutation who received anthracycline/taxane-based neo-adjuvant chemotherapy had pathologic complete response (pCR). The pCR of BRCA1 and BRCA2 mutation carriers were $38.3 \%$ [95\% Cl, $26 \%-55 \%]$ and $66 \%$ respectively, and $42.6 \%$ [ $95 \% \mathrm{Cl}, 29.2 \%-$ $56.8 \%$. The disease-free survival (DFS) and overall survival (OS) in the pCR group were much better than those in the non-pCR group, indicating a high $\mathrm{pCR}$ rate after therapy in BRCAmutated TNBC which is of prognostic value. Failure of TNBC patients to reach effective pCR in neo-adjuvant chemotherapy means poor prognosis. This theory is of interest to researchers in the study of the benefits of platinum drugs in treating tumors. A clinical trial has studied the feasibility of using homologous recombination deficiency (HRD) score to predict response of TNBC patients to platinumcontaining neo-adjuvant chemotherapy drugs [23]. Indeed, the efficacy of platinum drug therapy for TNBC is currently at the clinical trial phases, with emphasis on neo-adjuvant chemotherapies and chemotherapies in advanced TNBC patients [24,25]. However, this does not involve the trial phases of assisted therapy for TNBC after operation, which requires a large number of clinical data for verification.

\section{Epidermal or vascular endothelial growth factor receptor inhibitors}

Epidermal growth factor receptor (EGFR) and vascular endothelial growth factor receptor (VEGFR) are both classic structures of receptor tyrosine kinase (RTK); the former inevitably induces tumor cell proliferation, invasion, metastasis and angiogenesis by activating Ras-
Raf-MAPK, JNK and PI3 pathways [26, 27]. Overexpression of VEGR also affects these processes. The major inhibitors of EGFR are RTK inhibitor and anti-EGFR monoclonal antibody (mAb). These inhibitors could be potential targets for TNBC therapy [28].

In trials conducted by Baselga et al [29], a group of 173 patients with TNBC were randomly given either cisplatin combined with cetuximab or cisplatin alone. Compared with cisplatin group, the overall response rate (ORR) of the cisplatin + cetuximab group increased from $10.3 \%$ to $20 \%$. The median overall survival (OS) was in the range of 9.4 to 12.9 months, while the progression-free survival (PFS) increased from 1.5 months to 3.7 months. This showed that the addition of cetuximab to cisplatin could double ORR and prolong PFS and OS of TNBC patients. The novel targeted drug apatinib is an oral VEGFR which has been shown to exhibit certain effectiveness against TNBC [30]. Multicenter phase II studies revealed that the recommended dose of $750 \mathrm{mg}$ apatinib/day was better than 500 $\mathrm{mg} /$ day with respect to controlling the disease and adverse events. Research teams have examined VEGF-dependent biomarkers in patients using apatinib, to identify advanced breast cancer population sensitive to the drug $[30,31]$.

\section{Hepatocyte growth factor receptor inhibitor}

MET protein encoded by MET oncogene is a membrane-bound tyrosine kinase implicated in the formation and/or progression of TNBC. After activation by hepatocyte growth factor receptor (HGF), MET signaling pathway can induce the transformation of epithelia-mesenchymal transition (EMT) to augment cancer cell migration and invasion. Studies have confirmed that overexpression of c-Met can independently predict poor outcomes in breast cancer [32]. The progression of TNBC can be promoted by aberrant dysregulation of the receptor tyrosine kinase c-Met. A new report has shown that the common flavanol glycoside rutin is a potential lead molecule for preventing and controlling cMet-dependent breast malignancies [33]. Through bioassay-guided identification, rutin was found to inhibit breast cancer cell proliferation, migration and invasion. When rutin was injected into nude mouse at $30 \mathrm{mg} / \mathrm{kg}, 3$ times/week, the growth of MDA-MB-231/GFP orthotopic xenograft was significantly reduced in TNBC [33].

\section{$\mathrm{PI3K} / \mathrm{AKT} / \mathrm{mTOR}$ pathway inhibitor}

The viability, proliferation, amplification, migration, invasion and angiogenesis in several 
physiological and pathological processes are induced and mediated by the PI3K/AKT/mTOR pathway. The PI3K/AKT/mTOR pathway is overly activated in TNBC [34]. In a recent phase I trial of mTOR inhibition at the University Texas MD Anderson Cancer Center [35], advanced TNBC patients were treated with rapamycin combined with liposomal doxorubicin and bevacizumab. The results showed objective RR of $21 \%$, complete response (CR) of $8 \%$, partial response of $13 \%$, and the data showed disease stability for at least 6 months in $19 \%$ patients, a $40 \%$ clinical benefit rate, while $74 \%$ of the patients had aberrant PI3K pathway.

A randomized trial tested liposomal doxorubicin, bevacizumab, and temsirolimus (DAT) or liposomal doxorubicin, bevacizumab, and everolimus (DAE) to identify aberrations in PI3K pathway, with a view to improving the possibility of targeting the pathway to enhance chemotherapy response [35]. Rhizomaamorphophalli has also been shown to be a potential targeting therapeutic agent for TNBC [35]. Studies by $\mathrm{Wu}$ et al [36] found, through flow cytometry analysis that MDA-MB231 cells treated with RhA were arrested at the $S$ phase, because RhA decreased the migration and invasion of MDA-MB-231 cells, so that $\mathrm{PISK} / \mathrm{AKT} / \mathrm{mTOR}$ pathway was inhibited. This suggests that RhA is a potential therapeutic candidate in the treatment of TNBC through the inhibition of the $\mathrm{PI} 3 \mathrm{~K} / \mathrm{AKT} / \mathrm{mTOR}$ pathway.

\section{Androgen receptor inhibitor}

The androgen receptor (AR) accounts for $10 \%$ of LAR subtypes of TNBC, which is driven by AR signal and is sensitive to AR retardants. In ER subtypes, ERa and ER $\beta$ are two particular receptors which are closely associated with aggressive phenotypes of breast cancer [37]. This novel study revealed that the TNBC cell line can express endogenous ER $\beta 2$ only, and that its expression increased in TNBC cells and decreased cell proliferation and invasion. The expression of ERß2also decreased the expression of prolyl hydroxylase 3 (PHD3) gene and is linked to increased hypoxia inducible factor $1 \alpha \quad(H I F-1 \alpha)$ protein. These findings elucidated the importance of ER $\beta 2$ in enhancing TNBC cell proliferation and invasion, and also revealed that modulation of ER $\beta$ and ER $\beta 1$ signaling contributes to the invasive characteristics of TNBC [37]. Enzalutamide is a new-style anti-androgen drug. A well-designed multi-center Simon's phase II clinical trial evaluated the efficacy of enzalutamide on ARpositive TNBC, and found that clinical benefit rate (CR or PR or stable disease) was $42 \%$ during a 16-week follow-up. The data showed that non-luminal AR subtype of TNBC had low level of AR expression, indicating that it benefited from the AR- targeted therapy [38].

Steroid hormones in the supernatant of MDAMB-453 and primary cancer-associated fibroblasts (CAFs) were used by Kikuchi et al to study the correlation between androgen synthetic enzymes and CAFs [39]. The results revealed that the expressions of $17 \beta \mathrm{HSD} 2,17 \beta \mathrm{HSD} 5$, and $5 \alpha$-reductase 1 were increased by CAFs. In addition, $17 \beta \mathrm{HSD} 2$ and $17 \beta \mathrm{HSD} 5$ in IL-6 were partially dependent on phosphorylated STAT3, while the induction of HGF-mediated 5areductase1 was linked to phosphorylated ERK, suggesting that intra-tumoral androgen metabolism in ER-negative breast cancer are regulated IL-6 and HGF. These findings are helpful as novel references in individualized targeted therapy.

\section{FINAL REMARKS}

The genotype classification of TNBC is necessary for understanding the biological characteristics and clinical manifestation, and for developing individualized drug targeting for TNBC. Comprehensive clinical studies have shown that cytotoxic chemotherapy is the main therapeutic approach to TNBC. Indeed, PARP inhibitor and platinum drugs are very effective for treating TNBC with BRCA mutation. Researchers consider that these are not limited to this patient, gene homologous recombination is taken into consideration in the hope of development of clinical practices; It would be a significant hallmark to demonstrate effectiveness and tolerance of $A R$ inhibitors, since $A R$ is a molecular target with high positive expression in TNBC. In all, more clinical trials of subgroups in larger populations with heterogeneity of TNBC subtypes should be particularly focused on, so as to improve TNBC prognosis. Due to crosslinking of TNBC and signaling pathways, the application of different combinations of individualized drug targets offers great hopes for the treatment of TNBC.

\section{DECLARATIONS}

\section{Conflict of Interest}

No conflict of interest associated with this work.

\section{Contribution of Authors}

We declare that this work was done by Xiao-tian Ma, Shou-hua Rong, Yu-chao Zhang, Li-ting Jia in this article and all liabilities pertaining to claims 
relating to the content of this article will be borne by the authors. All authors read and approved the manuscript for publication. Xiao-tian Ma conceived and designed the study, Shou-hua Rong and Yu-chao Zhang collected and analyzed the data, Li-ting Jia wrote the manuscript.

\section{REFERENCES}

1. Guo J, Gong G, Zhang B. Screening and identification of potential biomarkers in triple-negative breast cancer by integrated analysis. Oncol Rep 2017; 38(4): 2219-2228.

2. Jhan JR, Andrechek ER. Triple-negative breast cancer and the potential for targeted therapy. Pharmacogenomics 2017; 18(17): 1-6.

3. Choi JE, Kang SH, Lee SJ, Bae YK. Androgen receptor expression predicts decreased survival in early stage triple-negative breast cancer. Ann Surg Oncol 2015; 22(1): 82-89.

4. Bianchini G, Balko JM, Mayer IA, Sanders ME, Gianni L. Triple-negative breast cancer: challenges and opportunities of a heterogeneous disease. Nat Rev Clin Oncol 2016; 13(11): 674-690.

5. Marotti JD, de Abreu FB, Wells WA, Tsongalis GJ. TripleNegative Breast Cancer: next-generation sequencing for target identification. Am J Pathol 2017; 187(10): 21332138.

6. Bae MS, Park SY, Song SE, Kim WH, Lee SH, Han W, Park IA, Noh DY, Moon WK. Heterogeneity of triplenegative breast cancer: mammographic, US, and MR imaging features according to androgen receptor expression. Eur Radiol 2015; 25(2): 419-427.

7. Yu DD, Lv MM, Chen WX, Zhong SL, Zhang XH, Chen L, Ma TF, Tang JH, Zhao JH. Role of miR-155 in drug resistance of breast cancer. Tumour Biol 2015; 36(3): 1395-1401.

8. Lehmann $B D$, Jovanović $B, X i C$, Estrada $M V$, Johnson $K N$, Shyr $Y$, Moses HL, Sanders ME, Pietenpol JA.. Refinement of triple-negative breast cancer molecular subtypes: implications for neoadjuvant chemotherapy selection. Plos One 2016; 11(6): 1-22.

9. Ollier M, Radosevicrobin N, Kwiatkowski $F$, et al. DNA repair genes implicated in triple negative familial nonBRCA1/2 breast cancer predisposition. Am J Cancer Res 2015; 5(7): 2113-2126.

10. Liu X, Li H, Shao B, Wu J, Kong W, Song G, Jiang $H$, Wang $J$, Wan F. Identification of recurrent BRCA1 mutation and its clinical relevance in Chinese triplenegative breast cancer cohort. Cancer Med 2017; 6(3): 547-554.

11. Perou CM, Sørlie T, Eisen MB, et al. Molecular portraits of human breast tumours. Nature 2000; 406(6797): 747752.

12. Lehmann $B D$, Bauer JA, Chen $X$, Sanders $M E$, Chakravarthy $A B$, Shyr $Y$, Pietenpol JA. Identification of human triple-negative breast cancer subtypes and preclinical models for selection of targeted therapies. $J$ Clin Invest 2011; 121(7): 2750-2767.

13. Player A, Abraham N, Burrell K, Bengone IO, Harris A, Nunez $L$, Willaims $T$, Kwende $S$, Walls W. Identification of candidate genes associated with triple negative breast cancer. Genes Cancer 2017; 8(7-8): 659-672.

14. Burstein MD, Tsimelzon A, Poage GM, et al. Comprehensive genomic analysis identifies novel subtypes and targets of triple-negative breast cancer. Clin Cancer Res 2015; 21(7): 1688-1698.

15. De Vos $M$, Schreiber $V$, Dantzer $F$. The diverse roles and clinical relevance of PARPS in DNA damage repair: current state of the art. Biochem Pharmacol 2012; 84(2): 137-146.

16. Helleday $T$. The underlying mechanism for the PARP and $B R C A$ synthetic lethality: clearing up the misunderstandings. Mol Oncol 2011; 5(4): 387-393.

17. Evans KW, Yuca E, Akcakanat A, Scott SM, et al. A Population of heterogeneous breast cancer patientderived xenografts demonstrate broad activity of PARP inhibitor in BRCA1/2 wild-type tumors. Clin Cancer Res 2017; 23(21): 6468-6477.

18. Lee JM, Hays JL, Chiou VL, et al. Phase I/lb study of olaparib and carboplatin in women with triple negative breast cancer. Oncotarget 2017; 8(45): 79175-79187.

19. Rodler ET, Kurland BF, Griffin M, et al. Phase I study of veliparib (ABT-888) combined with cisplatin and vinorelbinein advanced triple-negative breast cancer and/or BRCA mutation-associated breast cancer. Clin Cancer Res 2016; 22(12): 2855-2864.

20. Kummar S, Chen A, Ji J, et al. Phase I study of PARP inhibitor $A B T-888$ in combination with topotecan in adults with refractory solid tumors and lymphomas. Cancer Res 2011; 71(17): 5626-5634.

21. Clark CC, Weitzel JN, O'Connor TR. Enhancement of synthetic lethality via combinations of ABT-888, a PARP inhibitor, and carboplatin in vitro and in vivo using BRCA1 and BRCA2 isogenic models. Mol Cancer Ther 2012; 11(9): 1948-1958.

22. Bignon L, Fricker JP, Nogues C, et al. Efficacy of anthracycline/taxane-based neo-adjuvant chemotherapy on triple-negative breast cancer in BRCA1/BRCA2 mutation carriers. Breast $J$ 2017; 5: 1-11.

23. Telli ML, Timms KM, Reid J, et al. Homologous recombination deficiency (HRD) score predicts response to platinum-containing neoadjuvant chemotherapy in patients with triple-negative breast cancer. Clin Cancer Res 2016; 22(15): 3764-3773.

24. Petrelli F, Coinu A, Borgonovo K, Cabiddu M, Ghilardi M, Lonati $V$, Barni $S$. The value of platinum agents as neoadjuvant chemotherapy in triple-negative breast cancers: a systematic review and meta-analysis. Breast Cancer Res Treat 2014; 144(2): 223-32.

25. Guan X, Ma F, Fan Y, Zhu W, Hong R, Xu B. Platinumbased chemotherapy in triple-negative breast cancer: a systematic review and meta-analysis of randomizedcontrolled trials. Anticancer Drugs 2015; 26(8): 894-901. 
26. Zhang $P$, Zheng Z, Ling L, et al. w09, a novel autophagy enhancer, induces autophagy-dependent cell apoptosis via activation of the EGFR-mediated RAS-RAF1MAP2K-MAPK1/3 pathway. Autophagy 2017; 13(7): 1093-1112.

27. Blanchard Z, Paul BT, Craft B, EIShamy WM. BRCA1IRIS inactivation overcomes paclitaxel resistance in triple negative breast cancers. Breast Cancer Res 2015; 17(1): 5.

28. Aeri Kim, Min Hye Jang, Soo Jung Lee, Young Kyung Bae. Mutations of the epidermal growth factor receptor gene in triple-negative breast cancer. J Breast Cancer 2017; 20(2): 150-159.

29. Baselga J, Gómez P, Greil R, et al. Randomized phase II study of the anti-epidermal growth factor receptor monoclonal antibody cetuximab with cisplatin versus cisplatin alone in patients with metastatic triple-negative breast cancer. J Clin Oncol 2013; 31(20): 2586-92.

30. Hu X, Zhang J, Xu B, et al. Multicenter phase II study of apatinib, a novel VEGFR inhibitor in heavily pretreated patients with metastatic triple-negative breast cancer. Int J Cancer 2014; 135(8): 1961-1969.

31. Hu X, Cao J, Hu W, et al. Multicenter phase II study of apatinib in non-triple-negative metastatic breast cancer. BMC Cancer 2014; 14: 820.

32. Yan S1, Jiao X, Zou H, Li K. Prognostic significance of CMet in breast cancer: a meta-analysis of 6010 cases. Diagn Pathol 2015; 10: 62.

33. Elsayed $H E$, Ebrahim $H Y$, Mohyeldin MM, Siddique $A B$, Kamal AM, Haggag EG, El Sayed KA. Rutin as A novel
c-Met inhibitory lead for the control of triple negative breast malignancies. Nutr Cancer 2017; 30: 1-16.

34. Cancer Genome Atlas Network. Comprehensive molecular portraits of human breast tumours. Nature 2012; 490(7418): 61-70.

35. Basho RK, Gilcrease M, Murthy RK, et al. Targeting the PI3K/AKT/mTOR pathway for the treatment of mesenchymal triple-negative breast cancer: evidence from a phase 1 trial of mTOR inhibition in combination with liposomal doxorubicin and bevacizumab. JAMA Oncol 2017; 3(4): 509-515.

36. Wu C, Qiu S, Liu P, Ge Y, Gao X. Rhizoma Amorphophalli inhibits TNBC cell proliferation, migration, invasion and metastasis through the PI3K/Akt/mTOR pathway. J Ethnopharmacol 2018; 211: 89-100.

37. Bialesova L, XU L, Gustafsson JÅ, Haldosen LA, Zhao $C Y$, Dahlman-Wright K. Estrogen receptor $\beta 2$ induces proliferation and invasiveness of triple negative breast cancer cells: association with regulation of PHD3 and HIF-1a. Oncotarget 2017; 8(44): 76622-76633.

38. Barton VN, D' Amato NV, Gordon MA, et al. Multiple subtypes of triple-negative breast cancer critically rely on androgen receptor and respond to enzalutamide in vivo. Mol Cancer Ther 2015; 14(3): 769-778.

39. Kikuchi K, McNamara KM, Miki Y, et al. Effects of cytokines derived from cancer-associated fibroblasts on androgen synthetic enzymes in estrogen receptornegative breast carcinoma. Breast Cancer Res Treat 2017; 166(3): 709-723. 